\title{
A Short Note on the Fast Evaluation of Dihedral Angle Potentials and their Derivatives
}

\author{
Pedro Gonnet
}

September 26, 2011

Dihedral angle potentials, which are used in many force fields for molecular dynamics simulations, model the energy of twisting a bond as a function of the angle $\phi_{i j k l}$ between the surfaces spanned by the particles $p_{i}, p_{j}$ and $p_{k}$, and $p_{j}$, $p_{k}$ and $p_{l}$ respectively.

The potential function may have several forms. In the AMBER [4], CFF [6], CHARMM [7], DREIDING [8], GROMOS [9] and SHAPES [1] force fields, the potential is defined as

$$
v_{1}(\phi)=K[1+\cos (n \phi-\delta)],
$$

where $K$ is the energy of the potential, $n$ its multiplicity and $\delta$ its phase shift. The multiplicity $n$ is an integer and $\delta$ is usually an integer multiple of $\pi$.

In the Unified Force Field (UFF) [11], the potential function is generalized as

$$
v_{2}(\phi)=K \sum_{k=0}^{n} c_{k} \cos (k \phi),
$$

or, in Desmond [2], as

$$
v_{3}(\phi)=K \sum_{k=0}^{n} c_{k} \cos \left(k \phi-\phi_{0}\right),
$$

where $\phi_{0}$ is the equilibrium dihedral angle.

The first (1) and third (3) forms can be converted to the second form (2) when $\delta$ and $\phi_{0}$ are integer multiples of $\pi$. For all other values of $\delta$ and $\phi_{0}$, all three forms generalize to the form

$$
v_{4}(\phi)=K \sum_{k=0}^{n}\left[a_{k} \cos (k \phi)+b_{k} \sin (k \phi)\right],
$$

where the coefficients $a_{k}$ and $b_{k}$ can be obtained by applying the equivalence

$$
\cos (\alpha \pm \beta)=\cos (\alpha) \cos (\beta) \mp \sin (\alpha) \sin (\beta) .
$$

In practice, the angle $\phi$ is obtained by computing its sine and/or cosine from the vectors $p_{i}-p_{j}, p_{k}-p j$ and $p_{l}-p_{k}$, and computing its inverse sine, cosine or tangent. In NAMD [10] and GROMACS [5], $\phi$ is computed using the 
inverse tangent and then inserted into (1). In Desmond, the sine and cosine are computed using vector calculus and (3) is evaluated by repeated application of the equivalence (5), thus requiring no evaluations of any trigonometric functions or their inverses.

The entire computation can, however, be drastically simplified. Assuming that we have computed $x=\cos (\phi)$ using vector calculus, we can then re-write (1)-(4) in terms of $x$ :

$$
\begin{aligned}
& v_{1}(x)=K\left[1+T_{n}(x) \cos (\delta)+U_{n-1}(x)\left(1-x^{2}\right)^{1 / 2} \sin (\delta)\right], \\
& v_{2}(x)=K \sum_{k=0}^{n} c_{k} T_{k}(x), \\
& v_{3}(x)=K \sum_{k=0}^{n} c_{k}\left[T_{k}(x) \cos \left(\phi_{0}\right)+U_{k-1}(x)\left(1-x^{2}\right)^{1 / 2} \sin \left(\phi_{0}\right)\right], \\
& v_{4}(x)=K \sum_{k=0}^{n}\left[a_{k} T_{k}(x)+b_{k} U_{k-1}(x)\left(1-x^{2}\right)^{1 / 2}\right],
\end{aligned}
$$

where $T_{k}(x)$ and $U_{k}(x)$ are the Chebyshev polynomials of the first and second kind which satisfy

$$
T_{k}(x)=\cos \left(k \cos ^{-1}(x)\right), \quad U_{k-1}(x)=\frac{\sin \left(k \cos ^{-1}(x)\right)}{\left(1-x^{2}\right)^{1 / 2}} .
$$

Using the derivatives of the Chebyshev polynomials

$$
\frac{d}{d x} T_{k}(x)=k U_{k-1}(x) \text { and } \quad \frac{d}{d x} U_{k-1}(x)=\frac{k T_{k}(x)-x U_{k-1}(x)}{x^{2}-1},
$$

we can write the corresponding derivatives of the potentials with respect to $x$ as

$$
\begin{aligned}
\frac{d}{d x} v_{1}(x) & =K\left[1+n U_{n-1}(x) \cos (\delta)+\left(2 x U_{n-1}(x)-n T_{n}(x)\right)\left(1-x^{2}\right)^{-1 / 2} \sin (\delta)\right] \\
\frac{d}{d x} v_{2}(x) & =K \sum_{k=0}^{n} c_{k} k U_{k-1}(x) \\
\frac{d}{d x} v_{3}(x) & =K \sum_{k=0}^{n} c_{k}\left[k U_{k-1}(x) \cos \left(\phi_{0}\right)+\left(2 x U_{k-1}(x)-k T_{k}(x)\right)\left(1-x^{2}\right)^{-1 / 2} \sin \left(\phi_{0}\right)\right] \\
\frac{d}{d x} v_{4}(x) & =K \sum_{k=0}^{n}\left[a_{k} k U_{k-1}(x)+b_{k}\left(2 x U_{k-1}(x)-k T_{k}(x)\right)\left(1-x^{2}\right)^{-1 / 2}\right] .
\end{aligned}
$$

Note that when $\delta$ and $\phi_{0}$ are integer multiples of $\pi$, the terms in $\sin (\delta)$ and $\sin (\phi)$ can be dropped. 
The advantage of this formulation is that the Chebyshev polynomials can be evaluated using the three term recurrence relations

$$
\begin{array}{rrrl}
T_{0}(x) & =1 & T_{1}(x)=x, & T_{k}(x)=2 x T_{k-1}(x)-T_{k-2}(x), \\
U_{0}(x)=1 & U_{1}(x)=2 x, & U_{k}(x)=2 x U_{k-1}(x)-U_{k-2}(x)
\end{array}
$$

i.e. in $2 n-2$ additions and $2 n-1$ multiplications to obtain all $T_{k}(x), U_{k}(x)$ for $k=0 \ldots n$. Alternatively, the polynomails $U_{k}(x)$ for odd/even $k$ can be computed as twice the sum of $T_{j}(x)$, for all odd/even $0 \leq j \leq k$. Furthermore, $v_{2}(x)$ and $\frac{d}{d x} v_{2}(x)$ (as well as $v_{3}(x)$ and $\frac{d}{d x} v_{3}(x)$ when $\phi_{0}$ is an integer multiple of $\pi$ ) can be evaluated concurrently using the Clenshaw algorithm [3].

Although the relation of such potentials to polynomials in $\cos (\phi)$ is not entirely new [12], the connection to Chebyshev polynomials provides an efficient and general recipe for their evaluation.

\section{Acknowledgments}

The author would like to thank John Pearson, Mark Richardson and Asgeir Birkisson for the many fruitful discussions on this and other topics. This work was supported by the Swiss National Science Foundation Individual Support Fellowships PBEZP2-127959 and PA00P2_134146.

\section{References}

[1] Viloya S. Allured, Christine M. Kelly, and Clark R. Landis. SHAPES empirical force field: new treatment of angular potentials and its application to square-planar transition-metal complexes. Journal of the American Chemical Society, 113(1):1-12, 1991.

[2] K.J. Bowers, E. Chow, Huafeng Xu, R.O. Dror, M.P. Eastwood, B.A. Gregersen, J.L. Klepeis, I. Kolossvary, M.A. Moraes, F.D. Sacerdoti, J.K. Salmon, Yibing Shan, and D.E. Shaw. Scalable algorithms for molecular dynamics simulations on commodity clusters. In $S C 2006$ Conference, Proceedings of the ACM/IEEE, page 43, 2006.

[3] C. W. Clenshaw. A note on the summation of Chebyshev series. Mathematical Tables and Other Aids to Computation, 9(51):118-120, 1955.

[4] Yong Duan, Chun Wu, Shibasish Chowdhury, Mathew C. Lee, Guoming Xiong, Wei Zhang, Rong Yang, Piotr Cieplak, Ray Luo, Taisung Lee, James Caldwell, Junmei Wang, and Peter Kollman. A point-charge force field for molecular mechanics simulations of proteins based on condensed-phase quantum mechanical calculations. Journal of Computational Chemistry, 24(16):1999-2012, 2003. 
[5] Berk Hess, Carsten Kutzner, David van der Spoel, and Erik Lindahl. GROMACS 4: Algorithms for highly efficient, load-balanced, and scalable molecular simulation. Journal of Chemical Theory and Computation, 4(3):435447, 2008.

[6] S. Lifson and A. Warshel. Consistent force field for calculations of conformations, vibrational spectra, and enthalpies of cycloalkane and n?alkane molecules. Journal of Chemical Physics, 49(11):5116-5129, 1968.

[7] A. D. MacKerell, D. Bashford, Bellott, R. L. Dunbrack, J. D. Evanseck, M. J. Field, S. Fischer, J. Gao, H. Guo, S. Ha, D. Joseph-McCarthy, L. Kuchnir, K. Kuczera, F. T. K. Lau, C. Mattos, S. Michnick, T. Ngo, D. T. Nguyen, B. Prodhom, W. E. Reiher, B. Roux, M. Schlenkrich, J. C. Smith, R. Stote, J. Straub, M. Watanabe, J. Wiórkiewicz-Kuczera, D. Yin, and M. Karplus. All-atom empirical potential for molecular modeling and dynamics studies of proteins. The Journal of Physical Chemistry B, 102(18):3586-3616, 1998.

[8] Stephen L. Mayo, Barry D. Olafson, and William A. Goddard. DREIDING: a generic force field for molecular simulations. The Journal of Physical Chemistry, 94(26):8897-8909, 1990.

[9] Chris Oostenbrink, Alessandra Villa, Alan E. Mark, and Wilfred F. Van Gunsteren. A biomolecular force field based on the free enthalpy of hydration and solvation: The GROMOS force-field parameter sets 53A5 and 53A6. Journal of Computational Chemistry, 25(13):1656-1676, 2004.

[10] James C. Phillips, Rosemary Braun, Wei Wang, James Gumbart, Emad Tajkhorshid, Elizabeth Villa, Christophe Chipot, Robert D. Skeel, Laxmikant Kal, and Klaus Schulten. Scalable molecular dynamics with NAMD. Journal of Computational Chemistry, 26(16):1781-1802, 2005.

[11] A. K. Rappe, C. J. Casewit, K. S. Colwell, W. A. Goddard, and W. M. Skiff. UFF, a full periodic table force field for molecular mechanics and molecular dynamics simulations. Journal of the American Chemical Society, 114(25):10024-10035, 1992.

[12] Tamar Schlick. A recipe for evaluating and differentiating $\cos \phi$ expressions. Journal of Computational Chemistry, 10(7):951-956, 1989. 